\title{
Electrically-tunable surface deformation of a soft elastomer
}

\section{Citation}

Shian, Samuel, and David R. Clarke. 2016. Electrically-Tunable Surface Deformation of a Soft Elastomer. Soft Matter 12, no. 13: 3137-3141. doi:10.1039/c6sm00090h.

\section{Published Version}

10.1039/c6sm00090h

\section{Permanent link}

http://nrs.harvard.edu/urn-3:HUL.InstRepos:34325446

\section{Terms of Use}

This article was downloaded from Harvard University's DASH repository, and is made available under the terms and conditions applicable to Open Access Policy Articles, as set forth at http:// nrs.harvard.edu/urn-3:HUL.InstRepos:dash.current.terms-of-use\#OAP

\section{Share Your Story}

The Harvard community has made this article openly available.

Please share how this access benefits you. Submit a story.

Accessibility 


\section{Electrically-Tunable Surface Deformation of a Soft Elastomer

\author{
Samuel Shian and David. R. Clarke
}

John A Paulson School of Engineering and Applied Sciences, Harvard University, 29 Oxford St., Cambridge, MA 02138, USA

The flat surface of a thin elastomer on a conducting substrate can be deformed by applying an electric field to a percolating network of metallic nanowires randomly dispersed over the surface. The magnitude of the fieldinduced surface undulations increases with the applied field and can locally be several times the diameter of the nanowires. Optical imaging indicates that the effect is reversible and the surface flatness recovers when the electric field is removed. It is found that it is the field-induced changes in surface morphology rather than the nanowires themselves that strongly scatter light. The optical effects could be exploited in functional devices including tunable privacy windows, displays, and camouflage. There is also the potential for tuning the adhesion of elastomers to other materials.

The surface morphology of elastomers and polymers can be altered in a number of different ways, for instance by exposing to a different chemical environment, applying an external force, by changing the temperature, or, for some materials, by applying to electric field. These are typically slow processes and the surface deformations produced are often irreversible. For instance, when PDMS is exposed to ionizing radiation, such as a gas plasma used to increase adhesion to its surface, it wrinkles in response to the shrinkage in the near-surface region. ${ }^{1,2}$ Similarly, swelling of a laterally confined polymer film exposed to a solvent, whether on a solid or liquid substrates, can create wrinkle patterns. ${ }^{3}$ Wrinkling also can be produced by exploiting large strain incompatibility between different materials, such as metallic films on an elastomeric surface, in response to changes in temperature. ${ }^{4,5}$ Recent works have used electric field to induce electromechanical instabilities, leading to deformation on the surface. ${ }^{6-10}$ In some cases, the deformation is quite fast, ${ }^{7,11}$ however the use of a highly conducting, thick liquid electrolyte as an electrode limits its practicality. In this work, we describe an alternative, more rapid as well as reversible process based on electric field controlled electrostatic interactions across the thickness of a thin sheet of elastomer. The magnitude of the resulting surface deformations can be controlled by varying the applied electric field. As an example of the utility of being able to control the surface morphology of an elastomer, we demonstrate that the reflectance and transmission of light incident on a tunable surface can be modulated.

During the course of our work utilizing percolative networks of SWCNT and silver nanowires as compliant, electrically conducting electrodes for dielectric elastomer actuators ${ }^{12}$ and energy harvesting systems ${ }^{13,14}$, we noticed that the surface deformed slightly when an electric field was applied to the electrodes. To investigate this observation systematically, we fabricated a simple layered structure consisting of a thin sheet of elastomer attached to a ITO coated soda-lime glass substrate with a thin layer of randomly oriented silver nanowires on top. The elastomer, a polyacrylate (3M VHB 4905) sheet of nominal thickness $500 \mu \mathrm{m}$, was first pre-stretched biaxially to $300 \%$ linear strain, producing a sheet 31 $\mu \mathrm{m}$ thick. The thinned elastomer sheet was then attached on a ITO-coated glass with care being taken to avoid trapping any air. Silver nanowires (AgNW) electrodes with target areal density of $78 \mathrm{mg} / \mathrm{m}^{2}$ were prepared from an ethanol suspension $(90 \mathrm{~nm}$ average diameter, 20-60 $\mu \mathrm{m}$ length, SLW-NW-90, Blue Nano, Charlotte, NC), cast through a Teflon filter and then transferred to the surface of a thin elastomer sheet ${ }^{12}$. The nanowire network was connected to a high-voltage source with the other electrode being the conducting substrate. With increasing voltage, the surface deformed as shown in the optical micrographs of Fig. 1 and in the confocal images of Fig. 2. 

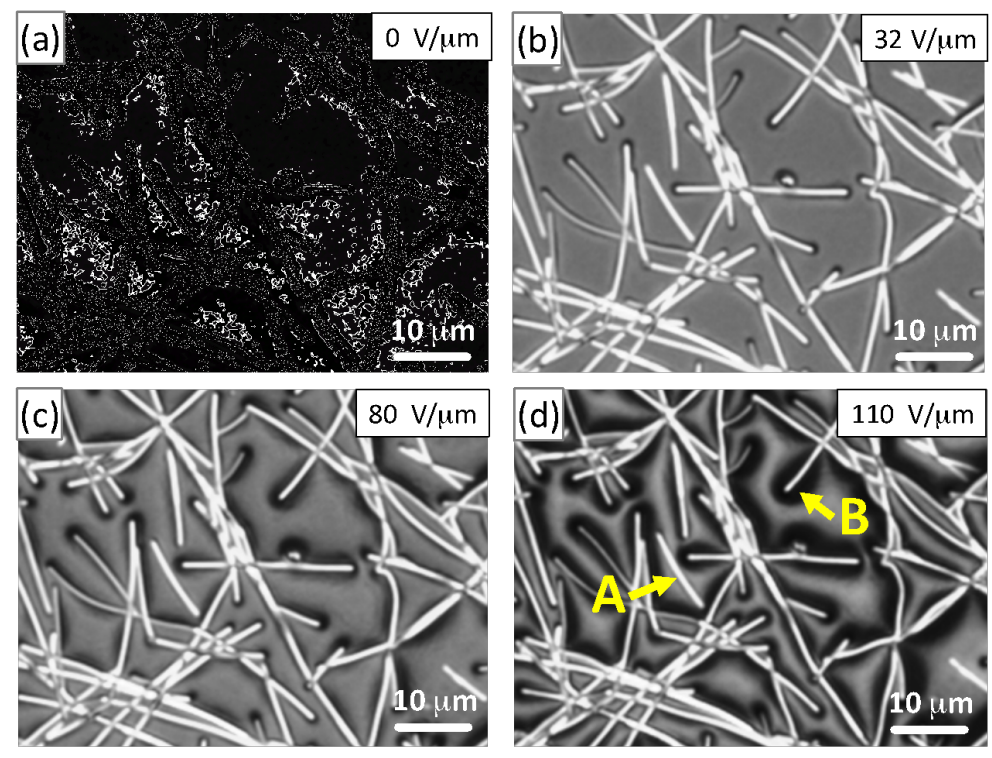

(e)

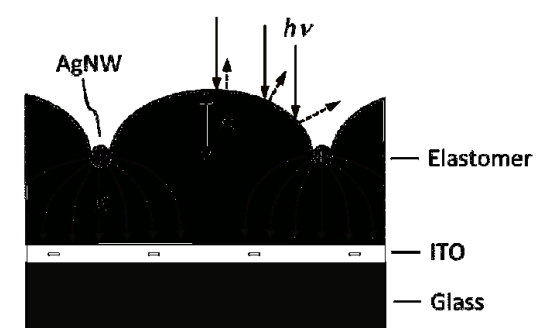

Fig. 1. (a) Bright field optical microscope image showing the spatial distribution of the silver nanowires and the surface of the elastomer in between. The areal density of nanowires is $\sim 78 \mathrm{mg} / \mathrm{m}^{2}$. (b) to (d) the same region at the indicated electric fields (nominal) showing the field-induced local deformations of the elastomer surface revealed by optical contrast change. (e) A schematic cross-section of the surface displacements and the electric field emanating from individual nanowires.

When connected to a voltage source the nanowire network charges and is attracted by electrostatic forces to the oppositely charged ITO electrode. For a uniformly distributed charge on the surface of a dielectric having a permittivity, $\varepsilon$, the force acting on the surface is the Maxwell stress tensor $\sigma$. In the absence of magnetic field, this can be expressed as ${ }^{15,16}$ :

$$
\boldsymbol{\sigma}=\varepsilon \mathbf{E E}-1 / 2 \varepsilon(\mathbf{E} \cdot \mathbf{E}) \mathbf{I}
$$

where $\boldsymbol{E}$ is the electric field, and $\boldsymbol{I}$ is the identity tensor. The direction of the Maxwell stress at any point on the surface is parallel to the direction of the local electric field, which is, in turn, perpendicular to the interface of the conductor and dielectric. This electric field is depicted by the lines emanating from the nanowires to the other electrode interface, as shown schematically in the Fig 1(e). At the interface of dielectric and nanowires, the normal force is $F_{n}=\boldsymbol{n} \cdot \boldsymbol{\sigma} \cdot \boldsymbol{n}=1 / 2 \varepsilon \boldsymbol{E} \cdot \boldsymbol{E}$, and the tangential force $F_{t}=\boldsymbol{t} \cdot \boldsymbol{\sigma} \cdot \boldsymbol{n}=0$, where $\mathrm{n}$ and $\mathrm{t}$ are the unit normal and tangential vectors to the interface of nanowires/elastomer, respectively. Because of the random distribution of nanowires in the plane of the surface, the electric field is not uniform over the surface but is highest immediately under the individual nanowires. Furthermore, as the cross section of the silver nanowires are distorted pentagons, ${ }^{17}$ there is a further enhancement of electric field around the edge of the nanowires. Nevertheless, the simpler case of the force between a charged cylindrical wire parallel to an infinite conducting plane and embedded in a uniform dielectric expresses the principal dependencies. For this case, the attractive force per unit length of wire, $F_{n} / l$, acting towards the conducting plane, can be written as ${ }^{18}$ :

$$
F_{n} / l=\frac{\pi \varepsilon}{4 a^{2}} \frac{\Phi^{2}}{\left.\cosh ^{-1}(H / R)\right]^{2}}
$$

where $\Phi$ is the potential, $H$ is the distance between the axis of wire and plane, and $R$ is the wire radius, and $a=1 / 2\left(H+\sqrt{H^{2}-R^{2}}\right)$. This attractive force deforms the elastomer locally altering its surface morphology. In the following experiments the electric field is reported as nominal values, defined as the applied voltage divided by the initial thickness of the elastomer, rather than the instantaneous thickness. 


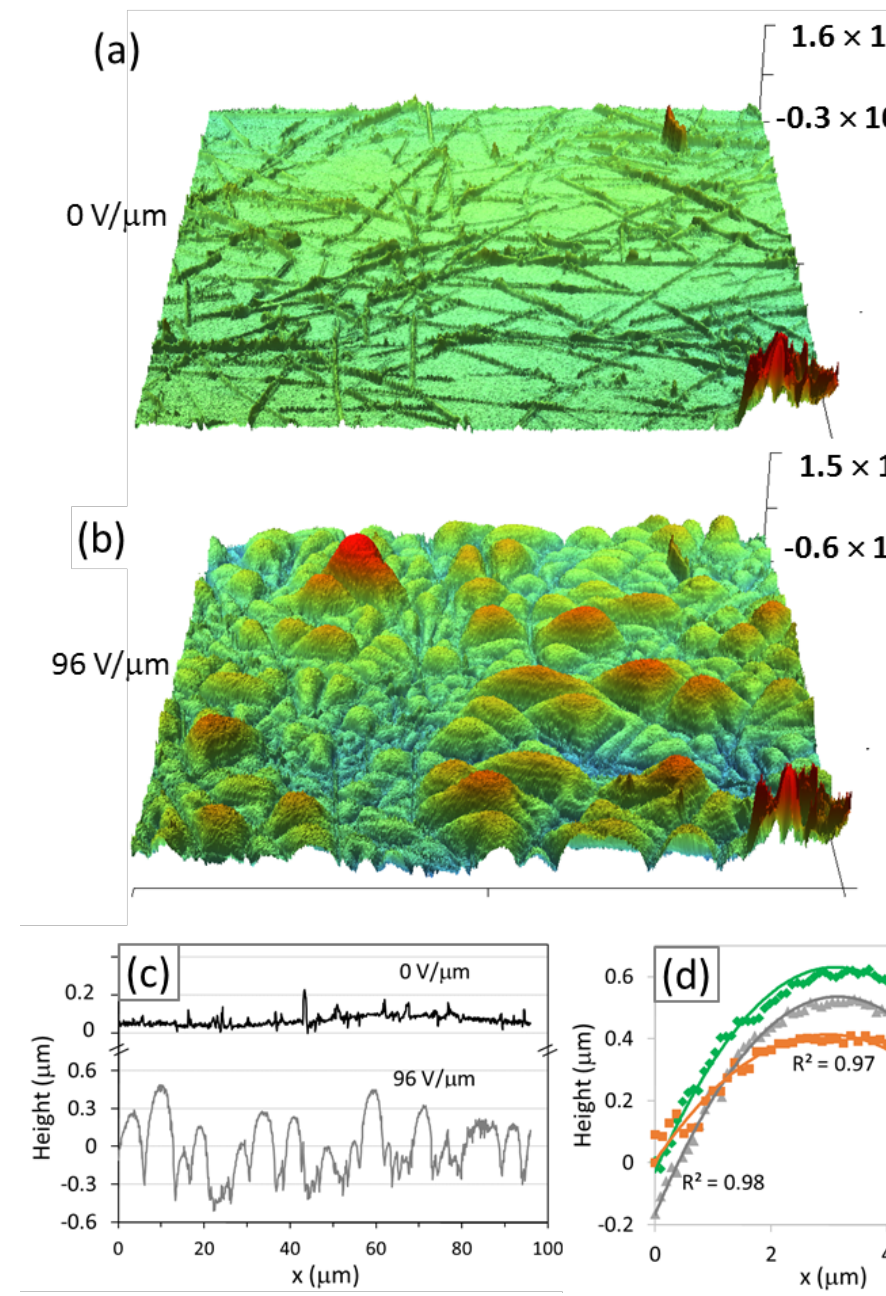

$1.6 \times 10^{-3}$

$-0.3 \times 10^{-3}$

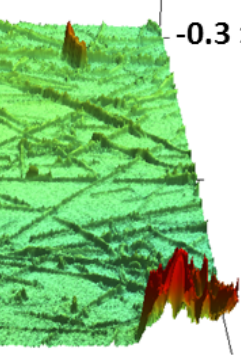

$1.5 \times 10^{-3}$

(b)

$-0.6 \times 10^{-3}$

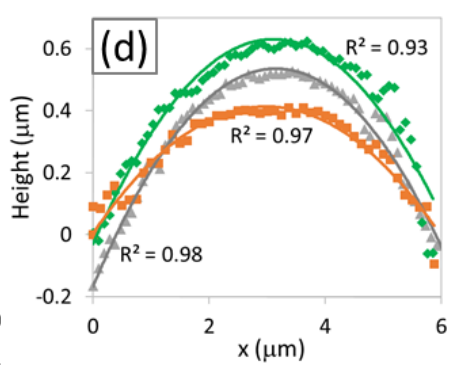

Fig. 2. Confocal microscopy maps of the nanowire electrode and the elastomer surface. (a) without any field and (b) at an applied electric field (nominal) of $96 \mathrm{~V} / \mu \mathrm{m}$. The scanned area is $96 \times 96 \mu \mathrm{m}^{2}$. (c) Line profiles show that the surface morphology is much rougher and in places can be several times the diameter of the silver nanowires (diameter 90nm) when the voltage is applied. The dips in the lower trace correspond to the locations of individual nanowires. (d) Detailed fitting of the surface bulges formed between two nearly parallel nanowires. These profiles can be fit reasonably well using parabolic equation (solid lines).

Unlike the nanowires, the ITO conducting surface is a continuous coating on a much thicker, solid, glass substrate. Consequently, the substrate imposes a mechanical constraint on the lateral expansion of the elastomer which, being an incompressible material, cannot change volume. So, when the nanowires are charged by applying a voltage their displacement towards the ITO coated glass substrate produces an average displacement of the elastomer between the nanowires in the opposite direction in order to conserve the overall volume of the elastomer. The displacement of the elastomer surface varies spatially because of the inhomogeneous distribution of the randomly distributed nanowires as can be seen in the bright field reflection optical microscopy comparing the images in Fig 1 before and during the application of electric field. Dark outlines along the length of the nanowires indicate that light is reflected at an angle away from the incident illumination by the local curvature of deformed elastomer surface. These dark outlines diminish away from the nanowires indicating that the surface has bulged upwards in between. Comparison of the individual images also shows that the lateral positions of the individual nanowires are unchanged as the electric field is applied. Another feature of the images is that as the applied electric field is increased, the optical contrast becomes more pronounced especially at the ends of the nanowires, as illustrated by the ' $B$ ' arrow in Fig $1 \mathrm{a}$. Also, unless the nanowires are close together, the optical contrast is symmetrical about the axis of the nanowires. A notable feature of the images is that the elastomer surface is not deformed in the immediate vicinity of individual nanowires that are not connected to the percolating network. These can be identified by the nanowires that are not outlined by a band of dark contrast, for instance, the one marked by ' $A$ ' arrow in Fig $1 \mathrm{~d}$. 

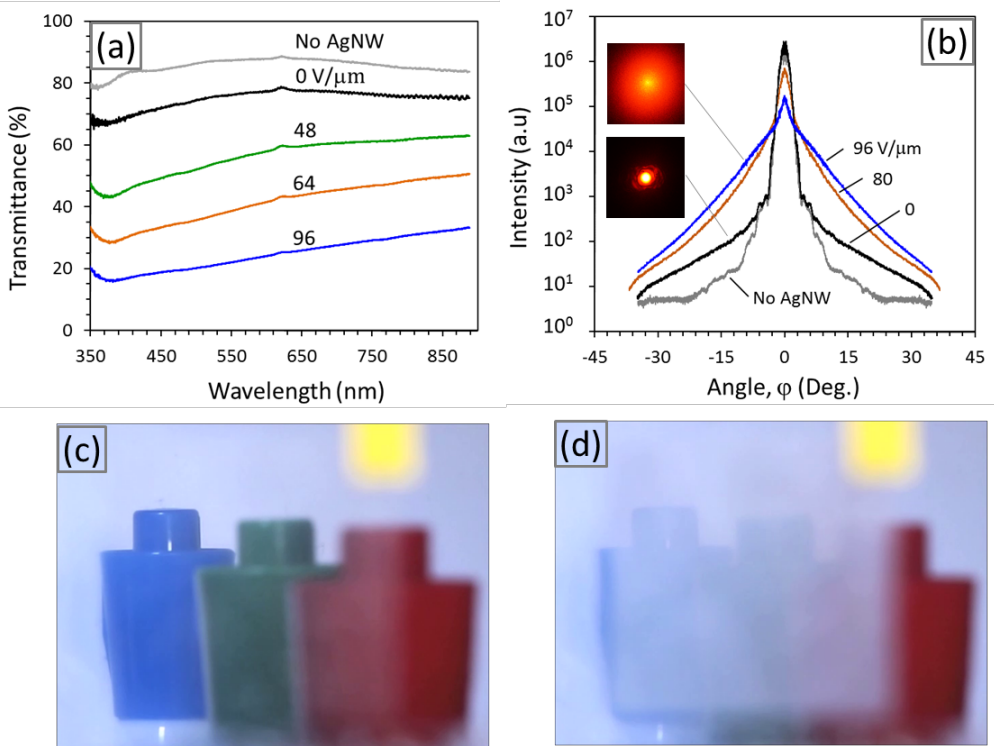

Fig. 3. (a) In-line spectral transmittance for different applied nominal field. There is a slight absorption centered at about $360 \mathrm{~nm}$ which is attributed to plasmon resonance in the silver nanowires. (b) Angular distribution of the transmitted laser light (632 $\mathrm{nm}$ ). The broadening is increased substantially as the surface deforms when the electric field is increased. This is also seen qualitatively in the recorded images in the insert. (c) A demonstration of light scattering is shown by the change in the clarity of the objects placed behind the sheet (c) before and (d) after the application of field of $130 \mathrm{~V} / \mu \mathrm{m}$. Upon removal of field, the sheet immediately returns to its initial, transparent condition. The electrode area is square, as shown by the white opaque area in (d) and is connected to an external power supply through the yellow tab in the upper right. The measurement setups for (a, b) and the real-time video of (c, d) are shown in Fig. S3 and Video 1 in the electronic supplementary information, respectively.

Confocal microscopy (Model VK-X200, Keyence Corp. with a $405 \mathrm{~nm}$ laser) was used to quantify the surface deformations as a function of applied electric field. The results are illustrated in Fig 2. Before an electric field is applied, the elastomer surface is relatively flat and the main contributions to the roughness are from the individual silver nanowires on the surface. Quantitative surface profiles indicate that the peaks have heights in the range of 80 to $170 \mathrm{~nm}$, which correspond to a single and two overlapping nanowires, respectively (Fig $2 \mathrm{c}$ ). With the application of an electric field, the morphology of the elastomer surface changes continuously, producing deep depressions at the individual nanowires and bulges in between the individual nanowires. The maximum peak-to-valley displacements were more than $600 \mathrm{~nm}$ for an applied nominal field of $96 \mathrm{~V} / \mu \mathrm{m}$ indicating that the nanowires are displaced towards the ITO electrode many times their diameter (Fig. 2d). The magnitude of the surface displacement also varies with position, depending on the spacing between adjacent percolating nanowires; relatively small vertical displacements occurred where the spacing between the nanowires was small whereas where their spacing was larger, the relative heights of the bulges were larger. Sampling of the surface profiles of the elastomer between two adjacent and nearly parallel nanowires indicate they are, to a good fit, parabolic. This is illustrated by the profiles in Fig. $2 \mathrm{~d}$.

Although the optical images, such as those in Fig. 1, reveal local variations in the surface as an electric field is applied as well as the association with individual nanowires, providing some physical insight, the random arrangement of the nanowires makes it difficult to establish the overall deformation of the surface. An alternative, more effective measurement of the overall response can be monitored from variations in the transmitted light with applied electric field. An example is shown in Fig. 3a using the optical arrangement in Fig S1(a) (supplementary materials). In this measurement, the combination of collimating lenses and the $50 \mathrm{~mm}$ spacing between sample and detector allows for the detection of the in-line transmittance and the rejection of most of the scattered rays. The data, Fig. 3(a), indicate that the majority of the incident illumination is transmitted diffusely as the electric field is increased. The broad dip in transmission in the $400-500 \mathrm{~nm}$ spectral range (Fig. 3a) is attributed to the excitation of the localized surface plasmon resonance in the silver nanowires. ${ }^{19}$

The angular dependence of the transmitted intensity was measured using a 2D detector in the arrangement shown in Fig S1(b) Supplementary information. A highly collimated He-Ne laser $(1 \mathrm{~mm}$ diameter) was shone through the sample and detected by a 2D CMOS sensor $(15.6 \times 23.5 \mathrm{~mm}$, model NEX$3 \mathrm{~N}$, Sony Corp.) placed on a translation stage, at a distance of $22 \mathrm{~mm}$ from the sample, to enable measurements up to large angles. Images were captured in RAW mode at different exposure times to allow for the large dynamic range of the intensities. The pixel intensities were extracted using Image J 
software ${ }^{20}$ and plotted as a function of radial distance from the center of the beam. After stitching the data together from multiple exposures and positions, the resulting angular dependence was determined. An example is shown in Fig. 3b, which compares the angular scattering from the surface roughness of the elastomer, the additional scattering from the silver nanowires and the scattering when electric fields of 80 and $96 \mathrm{~V} / \mu \mathrm{m}$ are applied. The data plotted in Fig 3b clearly shows a broadening of the angular distribution in transmitted intensity as the electric field is increased. In the absence of the silver nanowires, the angular distribution is the intrinsic angular width of the laser beam and most of its intensity is confined within $\pm 5^{\circ}$. There are some weak diffraction peaks around the shoulder of the center beam, which originate from multiple interferences between the neutral density filters and the elastomer layers. When an electric field is applied, the angular profiles become much broader, and the intensity of at high angles increases at the expense of the intensity at the center beam. At a nominal electric field of $96 \mathrm{~V} / \mu \mathrm{m}$, the center (peak) intensity decreases by an order of magnitude, while the intensity at high angles increases by more than an order of magnitude out to $\sim 15$ degrees. The integrated intensity of the peak $\left(<5^{\circ}\right)$ represents the in-line transmission in the Fig $3(\mathrm{a})$. Visually, the broadening of the transmitted beam is evident from the photographs of the transmitted laser beam shown in the inset in Fig 3b. A demonstration of a tunable light diffuser is shown by placing objects behind an elastomer sheet as shown in Fig 3(c) and 3(d). These marker caps objects are placed at 110, 220 , and $330 \mathrm{~cm}$ from the sheet, where the left one is the furthest. In the absence of an electric field, the elastomer sheet is highly transparent and the objects can be clearly seen through it. After the application of an electric field of $130 \mathrm{~V} / \mu \mathrm{m}$, the sheet became hazy and the objects could no longer be clearly seen. The transparency of the sheet returns to its initial state upon removal of the field. The transition occurs relatively fast, at around $200 \mathrm{~ms}$, and is reproducible, as shown by the real-time Video 1 in the electronic supplementary information. The switching speed of the transition may be affected by several factors, which include charge re-distribution (or RC) time constant and viscoelastic of the elastomer. ${ }^{21}$ It is suspected however that the viscoelastic of acrylic elastomer could be the major limiting factor and therefore the switching speed may be improved using low viscoelastic elastomer, such as silicone.

The angular spread in transmitted intensity and its variation with electric field are due to a combination of different mechanisms. One is the optical scattering from the individual nanowires, a form of Rayleigh-Gans scattering, ${ }^{22}$ that scales with their shape and diameter. A second contribution is optical interference between nanowires, in particular in those regions where the nanowires are close together, a configurational dependent scattering. A third possible contribution is the refraction of the incident illumination by the local variations in the surface morphology. As the optical and confocal images indicate that the lateral positions of the nanowires do not change with the applied electric field, it can be concluded that the first two contributions cannot significantly affect the increase in angular scattering with electric field. Consequently, the refraction term, which does depend on the electric field induced force on the nanowires, is the major contribution to the angular broadening of the transmitted beam. Although the shape of the elastomer surface in the immediate vicinity of the nanowires is unknown because of the finite resolution of the confocal images, the shape at a distance a few wavelengths away can be approximated, as shown from the confocal images, by a parabolic surface. Simple ray tracing based on the refraction and reflection from parabolic surfaces, assuming no diffraction, leads to angular broadening that increases as the amplitude of the parabolic bulge increases. This is consistent with the observed angular broadening. In this simple model, however the maximum spread angle of the rays is determined by the maximum curvature of the parabola. Between these limits, the shape of the angular profile is an inverted projection of the parabolic bulge. Beyond this maximum angle, rather than decaying monotonically as is observed in Fig $3 b$, the intensity of refracted rays drops sharply to zero, except when the amplitude of bulge is high enough that multiple refraction occurs, spreading the rays to higher angle.

Quantitative modeling, taking into account diffraction effects and features sizes, was performed based on solving Maxwell's equations using the finite difference time-domain software (FDTD Solutions v8.12, Lumerical Solution Inc., Canada). The simulation cell is shown in Fig. 4(a) and consists of parallel nanowires, diameter $R$, with a lateral spacing, $L$, with the surface deformation in between given by a parabolic function $\left(f=b x^{2}\right)$ with an amplitude, $A$, that depends on the magnitude of the applied electric field. (In principal, as the force on an individual nanowire is known from equation 2, the resulting surface shape can be computed. However, this is difficult because of electro-mechanical coupling between the local electrostatic attraction and the mechanical response of the elastomer. This is further complicated by the fact that the elastomer is a hyper-elastic solid so the coupling is intrinsically nonlinear.) 

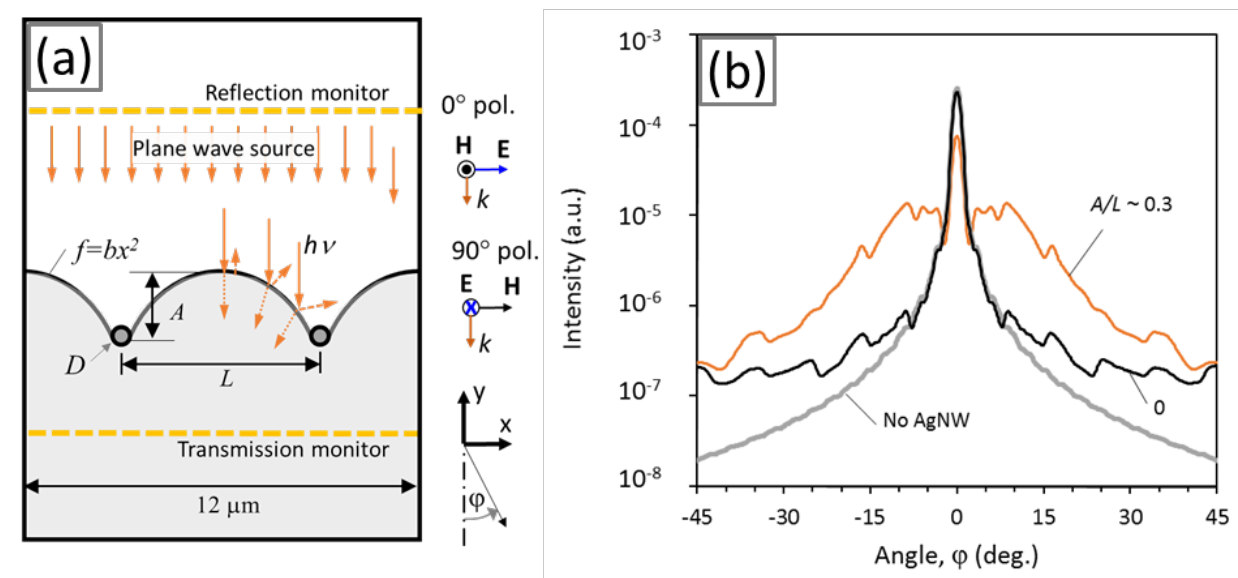

Fig. 4. (a) Schematic of the in FDTD 2D-simulation setup used to calculate angular distribution profile from a regularly spaced, $L$, parallel nanowires with diameter $D$, and surface undulations with amplitude $A$. (b) The results of simulation show the transmission as a function of spread angle, $\varphi$. The transmission is averaged from 4 different spacing $(1 \mu \mathrm{m}, 2 \mu \mathrm{m}, 6 \mu \mathrm{m}$, and 12 $\mu \mathrm{m})$ and each at similar relative bulge amplitudes $(A / L \sim 0.3)$

The left and the right sides of the simulation box are periodic boundaries while the top and bottom sides are perfectly absorbing boundaries. The width of the simulation box is fixed at $12 \mu \mathrm{m}$, which may contain one or multiple periodic structures, depending on the nanowire spacing $L$. The optical properties of the elastomer were assumed to be the same as those of silica $\left(\mathrm{SiO}_{2}\right)$. The optical data of silver and $\mathrm{SiO}_{2}$ were taken from Palik. ${ }^{23}$ Power-frequency monitors, positioned $500 \mathrm{~nm}$ below, and $2.5 \mu \mathrm{m}$ above, the center of the nanowires, are used in the simulation to determine the transmission and reflection at each wavelength. The angular intensity of the light is calculated using far-field projection from Poynting vectors that pass through the monitors. ${ }^{24}$ To smooth out the diffraction effects due to the periodicity in the simulation cell, the angular intensity profile was averaged over 150 profiles from different wavelengths in the visible range $(400-900 \mathrm{~nm})$. This averaging removes all the diffraction minima, except for the first minima that separate the zeroth and first diffraction orders. The simulation for each geometrical setup was performed twice, using $0^{\circ}$ and $90^{\circ}$ polarization light source and the results are averaged to represent non-polarized light source. The simulated angular scattering profiles for two different spacing $(L=2 \mu \mathrm{m}$ and $6 \mu \mathrm{m})$ of nanowires are shown in Fig. S3 (in the Supplementary Information). Finally, the angular intensity is averaged from four different spacing of nanowires from 4 different spacing $(L=1 \mu \mathrm{m}, 2 \mu \mathrm{m}, 6 \mu \mathrm{m}$, and $12 \mu \mathrm{m})$ with each at similar relative bulge amplitudes $(A / L \sim$ 0.3 ), as shown in Fig. 4(b). In the final averaging it was assumed that the area fraction of the nanowires was unchanged. In these averaged simulation profiles, the in-line transmittance is represented by the center region, which is peaked at $0^{\circ}$. All the intensity at higher angles is due to scattering and refraction.

Although approximate, the comparison in Fig. 4b exhibits many of the essential features of the observed optical scattering in Fig. 3b. This is despite neglecting several affects, such as due to the initial shape of the laser beam, spatial variations in the surface deformation, intrinsic roughness of the surface before actuation, the deformation around the ends of the nanowires, as well as the existence of the second air-elastomer interface at the exit surface. The simulations show that as the amplitude of the surface deformation increases, the intensity of diffused transmittance increases, accompanied by a decrease in the in-line transmittance. A similar trend is also observed in the reflected beam (shown in Fig S3 in the Supplementary information) although the overall reflected intensity is more than an order of magnitude less. The simulations show general agreement with experiment in that the silver nanowires causes scattering at large angles, above $10^{\circ}$ (compare Fig $3 \mathrm{~b}$ and $4 \mathrm{~b}$ ). Such scattering is more intense on the reflection side, as expected for metallic structures. In both cases, the intensity of the scattered beam increases as the concentration of nanowire increases. Comparison of the simulations in the absence of nanowires or using nanowires having the optical properties of glass instead of silver, indicate that the metallic contribution of the silver nanowires to the scattering is relatively constant, regardless to the magnitude of the surface deformation (Fig S2 in the Supplementary Information). At large deformations, the majority of the scattering in transmission is caused by the bulge geometry, rather than by the silver nanowires. 


\section{Summary}

We have shown that when a percolative network of nanowires on a soft elastomer is electrically charged it is attracted to an underlying conductor causing a spatially non-uniform surface deformation of the elastomer dielectric. The local surface displacements increase with electric field and can be many times the diameter of the nanowires. The field-induced surface undulations also strongly scatter visible light leading to an angular broadening of the transmitted light that increases with applied electric field. These deformation effects are fully reversible. Practical applications of the field-induced surface deformation include tunable privacy windows, smart glass, projector screens, displays, and camouflage. Outside of optics, the ability to electrically tune the surface deformation may be potentially useful in areas such haptics, adhesion controls, and functional surfaces.

\section{Acknowledgments}

This work was supported by the National Science Foundation through grant CMMI-1333835 and in part by the MRSEC program of the National Science Foundation under Award number DMR 14-20570. The authors would like to thank William Peabody of Keyence Corp. for assistance with the confocal microscopy and Peter Kjeer for facilitating the measurements.

\section{References}

1 J. Y. Chung, J. P. Youngblood and C. M. Stafford, Soft Matter, 2007, 3, 1163.

2 D. B. H. Chua, H. T. Ng and S. F. Y. Li, Appl. Phys. Lett., 2000, 76, 721.

3 E. P. Chan, E. J. Smith, R. C. Hayward and A. J. Crosby, Adv. Mater., 2008, 20, 711-716.

4 N. Bowden, S. Brittain and A. Evans, Nature, 1998, 393, 146-149.

5 J. Kim and H. H. Lee, J. Polym. Sci. Part B-Polymer Phys., 2001, 39, 1122-1128.

6 D. Van Den Ende, J. D. Kamminga, A. Boersma, T. Andritsch and P. G. Steeneken, Adv. Mater., 2013, $25,3438-3442$.

7 Q. Wang, L. Zhang and X. Zhao, Phys. Rev. Lett., 2011, 106, 118301.

8 N. Arun, A. Sharma, P. S. G. Pattader, I. Banerjee, H. M. Dixit and K. S. Narayan, Phys. Rev. Lett., 2009, 102, 1-4.

9 N. Wu and W. B. Russel, Nano Today, 2009, 4, 180-192.

10 E. Schaffer, T. Thurn-Albrecht, T. P. Russell and U. Steiner, Nature, 2000, 403, 874-877.

11 B. Xu and R. C. Hayward, Adv. Mater., 2013, 25, 5555-5559.

12 S. Shian, R. M. Diebold, A. McNamara and D. R. Clarke, Appl. Phys. Lett., 2012, 101, 061101.

13 J. Huang, S. Shian, Z. Suo and D. R. Clarke, Adv. Funct. Mater., 2013, 23, 5056-5061.

14 S. Shian, J. Huang, S. Zhu and D. R. Clarke, Adv. Mater., 2014, 26, 6617-6621.

15 A. Dorfmann and R. W. Ogden, J. Elast., 2006, 82, 99-127.

16 Q. Wang, Z. Suo and X. Zhao, Nat. Commun., 2012, 3, 1157.

17 Y. Sun, Y. Ren, Y. Liu, J. Wen, J. S. Okasinski and D. J. Miller, Nat. Commun., 2012, 3, 971.

18 R. Fitzpatrick, Maxwell's Equations and the Principles of Electromagnetism, Jones \& Bartlett Learning, 2008.

19 P. Spinelli and A. Polman, ACS Nano, 2012, 12, 3138-3144.

20 W. S. Rasband, ImageJ, U.S. National Institutes of Health, Bethesda, Maryland, USA, http://imagej.nih.gov/ij/, 2015.

21 L. Maffli, S. Rosset, M. Ghilardi, F. Carpi and H. Shea, Adv. Funct. Mater., 2015, 25, 1656-1665

22 H. C. van de Hulst, Light Scattering by Small Particles, Dover, 1957.

23 E. D. Palik, Ed., Handbook of Optical Constants of Solids, Academic Press, Burlington, 1997.

24 FDTD Solution Knowledge Base (User manual), Lumerical Solutions, Inc, 2015. 


\section{Electronic Supplementary Information}

\section{Electrically-Tunable Surface Deformation of a Soft Elastomer}

Samuel Shian and David R. Clarke

\section{Additional Experimental Details}

The in-line spectral transmittance was measured from $400 \mathrm{~nm}$ to $800 \mathrm{~nm}$ using an Ocean Optics spectrometer with a halogen light source together with a pair of collimators to direct the light entering and exiting the device. The setup is shown in Fig. S1(a).

The angular dependence of the transmitted intensity was measured using a 2D detector in the arrangement shown in Fig. S2(b). A collimated He-Ne laser $(\sim 1 \mathrm{~mm}$ diameter $)$ was shone through the sample and detected by a 2D CMOS sensor $(15.6 \times 23.5 \mathrm{~mm}$, model NEX-3N, Sony Corp.) placed on a translation stage, at a distance of $22 \mathrm{~mm}$ away from the device. By translating the sensor, the intensity could be measured up to large angles than possible with the sensor in a fixed position. Captured images were sticthed together and processed using ImageJ software to generate intensity as function of radial distance from the center of the beam.

Examples of simulated scattering profile from a parallel aray of nanowires at a constant spacing of $2 \mathrm{um}$ is shown in Fig. S2 and Fig. S3. In these figures, the angular intensity profile was averaged over 150 profiles obtained at different wavelengths in the visible range $(400-900 \mathrm{~nm})$. The intensity distibution in Fig. S2 were plotted in linear fashion to enhance the difference between the curves. In Fig. S2, the intensities are compared for silver nanowires, nanowires made of silica and where the nanowires are absent. 
(a)

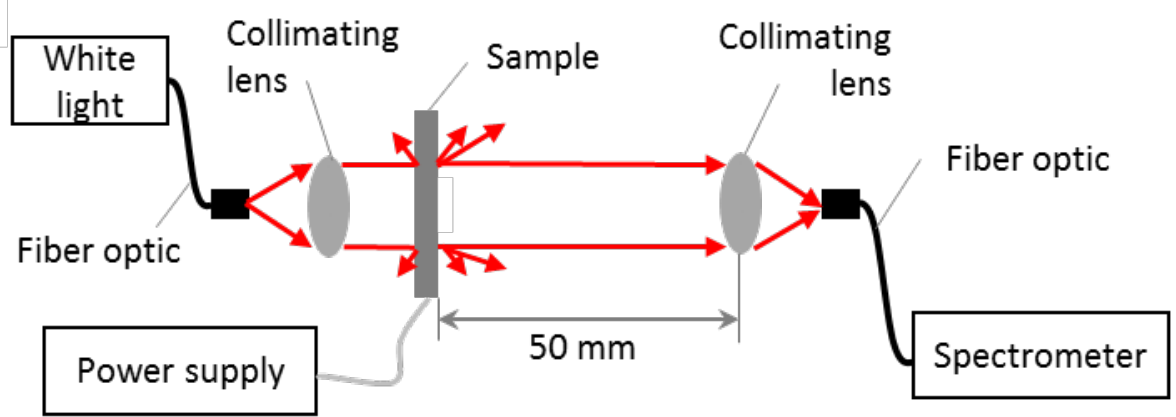

(b)

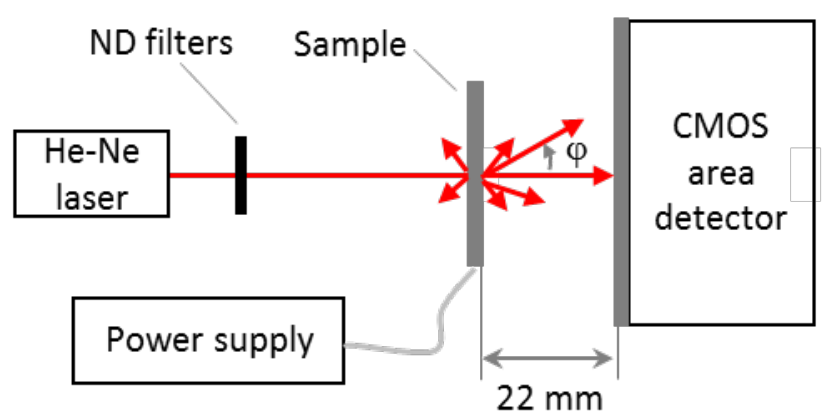

Fig. S1. Schematic of the arrangement for measuring the (a) direct transmittance and (b) angular broadening of the transmitted light. 

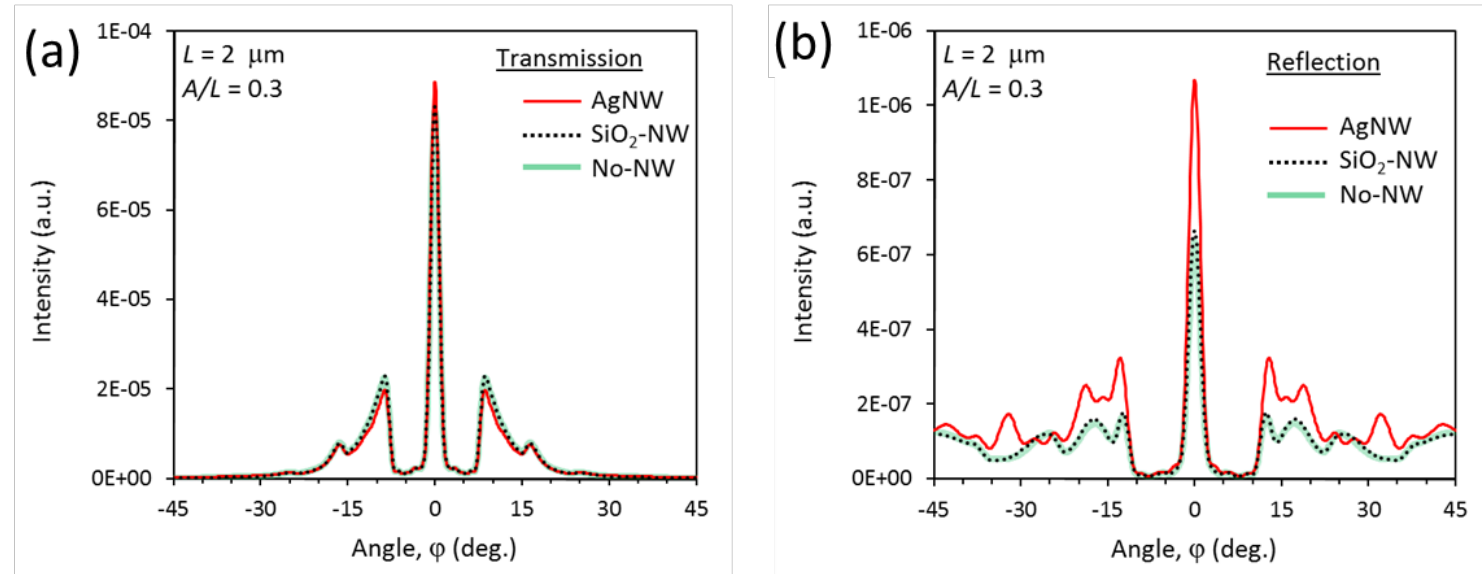

Fig. S2. Comparison of simulated scattering profile in absent of nanowires, just the bulge, (thick green line), with nanowire made of silver (red line), and nanowire made of glass (dotted line) are shown for (a) in transmission and (b) in reflection. At a relatively high bulge amplitude $A / L=0.3$, the scattering from metallic silver structure is negligible in the transmission and more pronounced in the reflection.
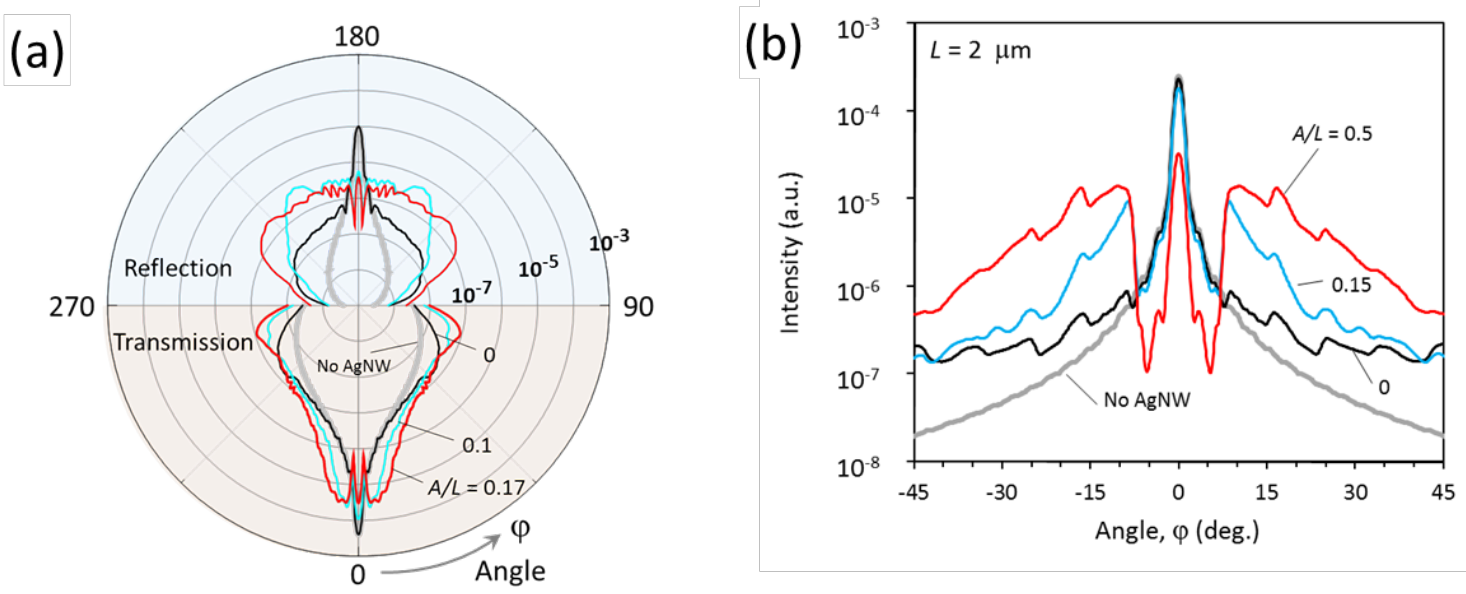

Fig. S3. Example of simulated scattering profile at (a) spacing at $\mathrm{L}=6 \mu \mathrm{m}$ and (b) $\mathrm{L}=2 \mu \mathrm{m}$. Note that in (a) both transmission and reflection are shown in a polar plot while in (b) only shows the angular dependence in transmission. 